\title{
New Insight in Recurrent Ovarian Torsion Managements" In A 12 Years Old Girl with Simple Cyst in Polycystic Ovary
}

\author{
Niyousha Shirsalimi ${ }^{1}$ and Shamim Pilehvari ${ }^{2,3 *}$ \\ ${ }^{1}$ Department of Medicine School, Hamadan University of Medical Sciences, Iran \\ ${ }^{2}$ Endometrium and Endometriosis Research Center, Hamadan University of Medical Sciences, Iran \\ ${ }^{3}$ Department of Obstetrics and Gynecology, Medicine School, Hamadan University of Medical Sciences, Iran
}

*Corresponding author: Shamim Pilehvari, Endometrium and Endometriosis Research Center, Hamadan University of Medical Sciences, Iran and Department of Obstetrics and Gynecology, Medicine School, Hamadan University of Medical Sciences, Iran.

To Cite This Article: Niyousha Shirsalimi, Shamim Pilehvari. New Insight in Recurrent Ovarian Torsion Managements" In A 12 Years Old Girl with Simple Cyst in Polycystic Ovary. Am J Biomed Sci \& Res. 2021 - 13(6). AJBSR.MS.ID.001922. DOI: 10.34297/AJBSR.2021.13.001922.

Received: 眥 July 23, 2021; Published: 觜 August 10, 2021

\begin{abstract}
Ovarian torsion is a rare but significant cause of acute abdominal pain in women. Commonly, patients present with the sudden onset of severe lower abdominal pain with progressive quality over hours. This condition is usually accompanied by decreased blood return from ovaries which leads to swelling and congestion of them. Abdominopelvic ultrasonography with color Doppler analysis is the first choice to diagnosis and estimating impairment of blood flow in ovaries. Quick diagnosis is required to protect the ovaries from necrosis and save the normal function.

In our case ,the treatment we performed after three time of recurrent ovarian torsion surgery ,was to wait and follow-up the patient continuously. In conclusion ,the last ultrasound which was performed 3 months after the last surgery showed that ,the size of the ovary returned to normal and the presence of blood flow and Antral follicle counts(AFC),indicates maintenance of normal ovarian function.
\end{abstract}

\section{Introduction}

Ovarian torsion is a condition caused by the complete or partial rotation of the ovary around the supporting ligaments which in most cases leads to impaired ovarian blood supply by compressing of the ovarian circulation. This disorder is a medical emergency and one of the most common causes of gynecological surgery which can involve women of all ages, even fetuses and infants. However, according to studies, women between the ages of 20_50 are the most affected $[1,2]$. Despite of the diversity in clinical manifestations of ovarian torsion, the classic presentation of this disorder include: moderate to severe acute onset of pelvic pain which is often accompanied by nausea and vomiting, and in some cases fever [3,4]. According to reports, torsion is more common in the right ovary probably due to the longer infandibulopelvic ligament on the right side and the protection effect of the sigmoid intestine on the left side of the body which prevents the left ovary from moving and twisting [5]. After taking a complete history of the patient and clinical examination and performing basic laboratory tests, ultrasound is performed as the first line of diagnosis of ovarian torsion by para-clinic which can determine the morphology, size, density and vascular flow of the twisted ovary that is often larger and rounder than a normal ovary due to impaired vascular and lymph flow. Our case is a premenarchal girl who suffered from left ovarian torsion three times during three months.

\section{Case Report}

Our patient was a 12-year-old girl referred to our gynecology clinic with chief complains of abdominal pain and vomiting and nausea with more intensity in the lower quadrant. The clinical signs in physical examination revealed an abdomen tenderness 
and guarding, normal limits in vital signs based on her age. The laboratory investigations showed count elevated white blood cells up to $16.400 \mathrm{~mm} 3$. Other basic biochemical test results' such as platelets count, hemoglobin rate, Urine analysis, renal function, serum electrolytes all were normal. The pregnancy blood test was negative. An abdominopelvic ultrasound scan revealed a left echogenic ovary with two cysts (33.38 $\mathrm{mm}$ and $37.29 \mathrm{~mm}$ ), with dimension in $88.56 .63 \mathrm{~mm}$ and volume as $160 \mathrm{cc}$ but dimension and echogenicity of right ovary were normal. The patient was prepared for surgery by ovarian torsion as primary diagnosis so Pfannenstiel incision laparoscopic detorsion of left ovary was performed.

After stabilizing the patient, she was discharged from the hospital but two months later was registered to the clinic again with complaining of sudden, sharp and severe lower abdominal pain. Abdominopelvic ultrasound demonstrated enlarged left ovary which contained two cysts with 37.36 and $39.35 \mathrm{~mm}$ in diameter. Doppler ultrasound of left ovary didn't show blood flows, so the decision was made for emergency laparoscopy which revealed a congested fragile tissue, bulky and completely necrotic with dark color (Figure1) in left ovary. Additionally, we found a 6 times torsion of Round ligament which was untwisted but due to inflamed and bloody tissues, cystectomy was not performed. About 20 days after second surgery, she was registered again with acute abdominal pain, anorexia, nausea and vomiting as chief complaint. In ultrasound examination, two cystic with 40.36 and $37.35 \mathrm{~mm}$ in dimensions were detected at the posterior cul-de-sac, originating from the left adnexa. The patient went under unilateral detorsion surgery with suspension technique for her left ovary with diagnosis of ovarian torsion for the third time within three months. Compared with the previous surgery, the volume of the left ovary was reduced and seemed normal with pink color. The Round and Uteroovarian Ligaments were fixed with U-shaped sutures and patient was discharged with LD contraceptive medication and recovery period in the hospital. The patient passed three months regular followup with ultrasound after the last surgery. Surprisingly in the last abdominopelvic ultrasound, ovarian volume, vascular echo and number of antral follicular counts (AFC) were reported normal (Figure 2).
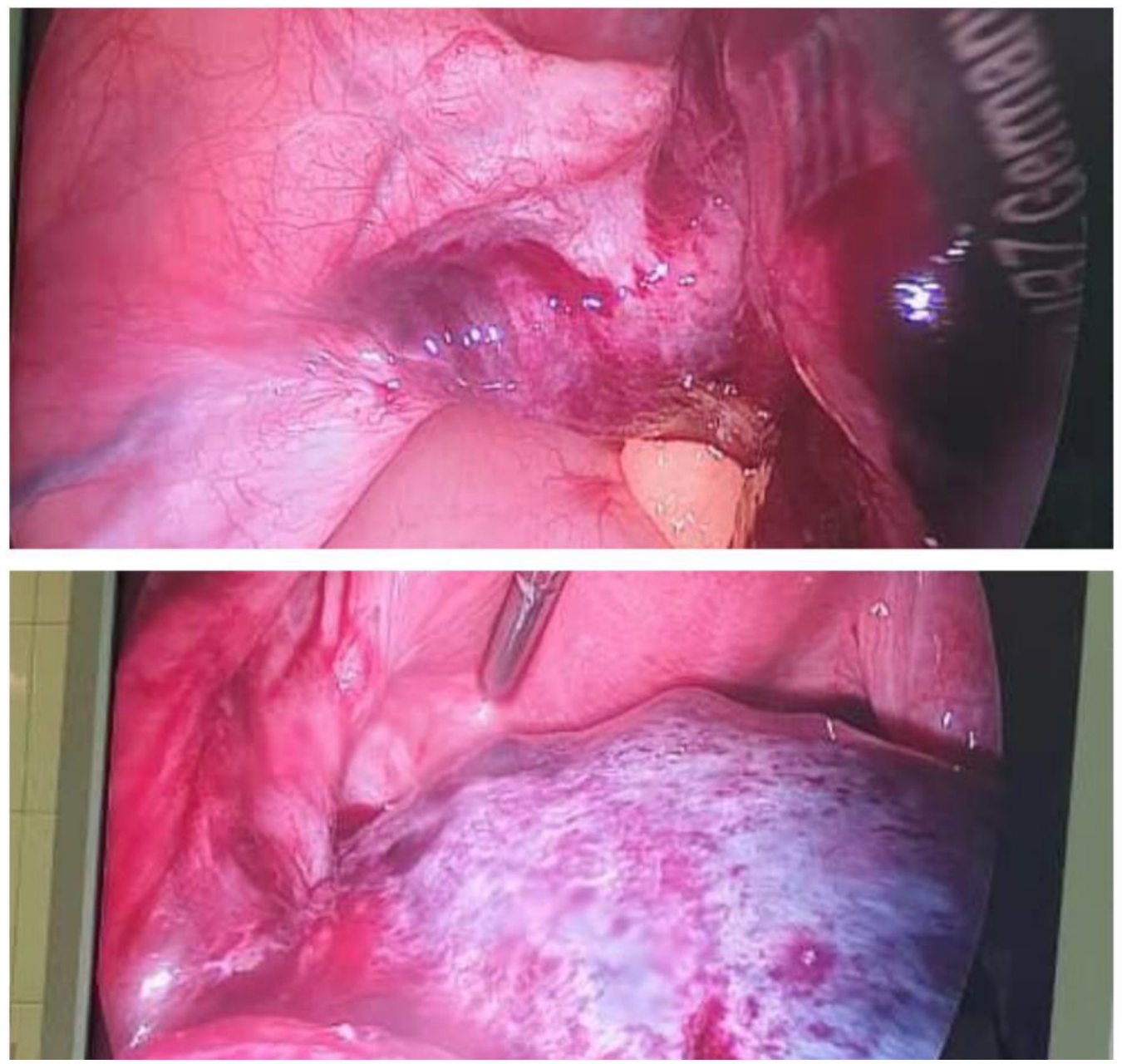

Figure 1: (intraoperative photo of recurrent twisted left ovarian in12-year-old girl with fragile and necrotic tissue in the second surgery). 


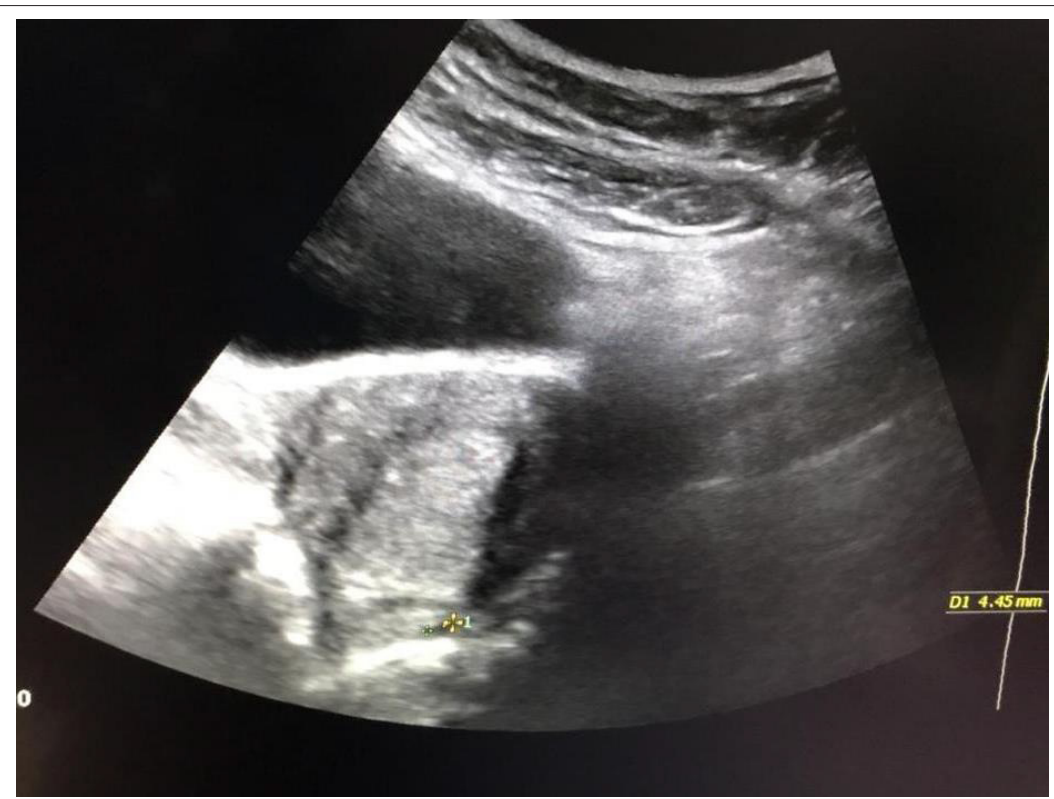

Figure 2: (ovarian volume, vascular echo and number of antral follicular counts (AFC) were reported normal in an ultrasound that was performed 3 months after the third surgery).

\section{Discussion}

The incidence of ovarian and adnexal torsion is unknown. Ovarian torsion can affect women of all ages, it can even occur in fetuses and Infants. However, the most commonly reported cases of ovarian torsion are the women in reproductive ages and sexually active who are between 20-50 years old [2]. Ovarian torsion can occur completely or partially, and according to studies, mostly on the right side. The longer utero ovarian ligament and the lack of a protective structure on the right side, such as the sigmoid colon which prevents ovarian motility on the left side, have been cited as possible causes in the articles [5]. The main risk factor for ovarian torsion is cystic or neoplastic mass. As a result, it significantly increases the risk of twisting in lower risk group, such as pre-monarchal girl ages [6,7]. Some data suggest that pregnancy, ovarian stimulation, tubal ligation, enlarged ovaries in polycystic ovary syndrome and history of abdominal surgery are associated with an increased risk of ovarian torsion, but the degree of these risks is uncertain $[2,8]$. Although, patients with torsion shows different clinical manifestations, but the most presentation of torsion include: abdominal pain, nausea, vomiting and nonmenstrual bleeding in rare cases $[3,4]$. Early diagnosis is very important to prevent severe consequences of torsion and preserve the function of ovaries and fallopian tubes [6]. For the approach to patient after taking the medical history and performing clinical examinations, basic laboratory tests are requested for the patient. In most scientific resources pelvic ultrasound is the first line choice of imaging study for patients which are suspected for ovarian torsion [9]. The most likely ultrasound findings in a patient with ovarian torsion are varied but commonly include: unilateral ovarian enlargement, peripheral masses and cysts free fluid in the pelvic cavity, large follicles and twisted ligaments. In scientific references laparoscopic and laparotomy surgery is recommended to confirm the definition diagnosis of ovarian torsion. However, a twisted ovary on initial examination may appear necrotic with black texture due to vascular and lymphatic congestion which were compressed by torsion. But researches and studies in this field around the word indicated that patients who underwent distortion surgery instead of salpingo-oopherectomy, after surgery and follow-up for several months, high percentages of patients showed normal follicle growth on ultrasound. The presence of antral follicular counts (AFC) on ultrasound of ovarian distortion indicates the survival of ovarian tissues with maintain normal function. during a study was performed on 40patients with ovarian torsion manifestation underwent ovarian detour surgery instead of complete ovarian resection in order to preservation the patient's reproductive function. intermediate period of hospilazation and the number of follicles was examined, as a result of this study expected post-operative treatment to detour the torsion is recommended to preservation ovarian function [10,11]. Another study was performed on 102 patients who underwent ovarian detorsion surgery, as a result, due to the benefits and function of the ovary after surgery instead of salpingo-oopharectomy, was suggested as the choice of treatment $[12,13]$. An important point is to emphasis medical intervention as soon as possible (under 36 hours), to prevent ovarian tissue necrosis and preserve its function.

Conclusion: ovarian torsion is a rare but urgency condition in gynecological surgery, which in many cases including our patient, the ovary that was initially black and necrotic with fragile and 
dysfunctional tissue, after detorsion and fixation surgery, with medical treatment and follow-up of the patient for the several months, the ovarian tissue became pink with normal function .in our case on last ultrasound examination AFC were observed. Therefore , that in same cases ,before performing aggressive treatment such as oophorectomy ,after detorsion surgical procedure ,with anticipatory care planning and follow-up the patient, give a chance to the damaged ovary to heal and tissue repair. this performance gives patients of reproductive ages, the opportunity to preserve their ovaries and fertility abilities to live perfectly normal.

\section{References}

1. Sanfilippo JS (2011) Surgery for benign disease of the ovary. The Linde's operative gynecology 480-775.

2. Tsafrir Z, Hasson J, Levin I, Solomon E, Lessing JB, et al. (2012) Adnexal torsion: cystectomy and ovarian fixation are equally important in preventing recurrence. Eur J Obstet Gynecol Reproductive Biol 162(2): 203-205.

3. Rousseau V, Massicot RI, Darwish AA, Sauvat F, Emond S, et al. (2008) Emergency management and conservative surgery of ovarian torsion in children: a report of 40 cases. J pediatr adolesc gynecol 21(4): 201-206.

4. Rossi BV, Ference EH, Zurakowski D, Scholz S, Feins NR, et al. (2012)The clinical presentation and surgical management of adnexal torsion in the pediatric and adolescent population. J pediatr adolesc gynecol 25(2): 109-113.
5. Vanessa N Weitzman, Andrea J DiLuigi, Donald B Maier, John C (2008) Nulsen Preventation of recurrent adnexal torsion. Fertil Steril 90(5): 2018.e1-e3.

6. White M, Stella J (2005) Ovarian torsion: 10-year perspective. Emerg Med Australas17(3): 231-237.

7. Kirkham YA, Lacy JA, Kives S, Allen L (2011) Characteristics and management of adnexal masses in a Canadian pediatric and adolescent population. J Obstet Gynaecol Can 33(9): 935-943.

8. Shah AA, Likes CE, Price TM (2009) Early polycystic ovary syndrome as a possible etiology of unexplained premenarcheal ovarian torsion. J pediatr adolesce gynecol 22(4): 265-269.

9. Huang C, Hong MK, Ding DC (2017) A review of ovary torsion. Tzu-chi Medical Journal 29(3): 143-147.

10. Oelsner G, Bider D, Goldenberg M, Admon D, Mashiach S (1993) Longterm follow-up of the twisted ischemic adnexa managed by detorsion. Fertil steril 60(6): 976-979.

11. Wang JH, Wu DH, Jin H, Wu YZ (2010) Predominant etiology of adnexal torsion and ovarian outcome after detorsion in premenarchal girls. Eur J pediatr surge 20(05): 298-301.

12. Cohen SB, Oelsner G, Seidman DS, Admon D, Mashiach S, et al. (1999) Laparoscopic detorsion allows sparing of the twisted ischemic adnexa. J Am Assoc Gynecol Laparosc 6(2):139-143. 